\title{
LISTA DE PUBLICAÇÕES DE HERMAN LENT
}

José Jurberg ${ }^{1}$

1.1934. Sobre dois novos gêneros da sub-família Trichostrongylinae Leiper, 1908, parasitos de Tinamus solitarius Vieill. Mem. Inst. Oswaldo Cruz 28 (2): 247-257, ests. 44-47, figs 1-12 (Com J.F. Teixeira de Freitas).

2. 1932. Capillaria cesarpintoi n.sp., parasita de Leimadophis poeci logyrus (Wied.). Mem. Inst. Oswaldo Cruz 28 (2): 263-265, est. 49, figs 1-3 (Com J.F. Teixeira de Freitas).

3. 1934. Capillaria amarali $\mathrm{n} . s p .$, parasita de Liophis miliaris miliaris (L.). Mem. Inst. Oswaldo Cruz 28 (2): 271-272, est. 53 figs 1-3 (Com J.F. Teixeira de Freitas).

4. 1934. Novo nematodeo da sub-família Heligmosominae Travassos, 1914, parasito de Agouti paca (L): Avellaria avellari n.g., n.sp., Mem. Inst. Oswaldo Cruz 28 (4): 577-583, ests. 77-78, figs 1-8 (Com J.F. Teixeira de Freitas).

5.1935. Sobre o macho de Capillaria auritae Trav., 1914 com uma nota a respeito da identidade dos gêneros Capillaria Zeder, 1800 e Eucoleus Duj., 1845. Rev. Med. Cir. Brasil 43 (4): 109-111 (Com J.F. Teixeira de Freitas).

6. 1935. Sobre uma nova espécie do gênero Trichostrongylus Looss, 1905. Rev. Med. Cir. Brasil 43 (6): 180-181, 1 fig. (Com J.F. Teixeira de Freitas).

7. 1935. Noções de technica helminthologica. O Hospital, Rio de Janeiro, 7 (7): 735-756 (Com J.F. Teixeira de Freitas.

8. 1935. Contribuição ao conhecimento da fauna helminthologica da Argentina. Graphidioides mazzai n.sp., parasito de Galealeucoblephara (Burm.). Rev. Med. Cir. Brasil 43 (7/8): 225-227, 1 est., figs 1-8 (Com J.F. Teixeira de Freitas).

9. 1935. Capillariinae de animais de sangue frio (Nematoda: Trichuroidea). Mem. Inst. Oswaldo Cruz 30 (2): 241-284,11 ests., figs 1-102 (Com J.F. Teixeira de Freitas).

10. 1935. Quelques variations et anomalies bursaires observées chez Viannaia viannai Trav., 1914 et Viannaia hamata Trav., 1914. Rev. Med. Cir. Brasil 43 (9): 253-258, figs 1-14 (Com J.F. Teixeira de Freitas).

11. 1935. Nota prévia sobre duas novas espécies do gênero Capillaria (Nematoda), e referência a novos hospedadores de helminthos conhecidos.

Rev. Med. Cir. Brasil 43 (10): 301-303 (Com J.F. Teixeira de Freitas).

12. 1935. Sobre a biologia, systematica e distribuição geographica do

1) Fundação Instituto Oswaldo Cruz, Caixa Postal 926, 21041-210 Rio de Janeiro, Rio de Janeiro, Brasil. 
Psammolestes coreodes Bergroth, 1911, encontrado em ninhos de aves no Brasil (Hemiptera, Triatomidae). Rev. Ent., Rio de Janeiro, 5 (4): 381-396, figs 1-6.

13. 1935. Sobre uma nova espécie do gênero Oswaldocruzia Travassos, 1917. Mem. Int. Oswaldo Cruz 30 (3): 379-386, figs, 1-7 (Com J.F. Teixeira de Freitas).

14. 1935. Sobre dois novos nematodeos parasitos da quica: Caluromys philander (L.). Mem. Inst. Oswaldo Cruz 30 (3): 535-542, figs 1-10 (Com J.F. Teixeira de Freitas).

15. 1935. Sobre as espécies do gênero Psammolestes Bergroth, 1911 (Hemiptera. Triatomidae). Ann. Acad. Brasil. Sci. 7 (4): 333-337), figs 1-5 (Com Cesar Pinto).

16. 1935. Duas novas espécies do gênero Capillaria Zeder, 1980, parasitas de Metachirops opossum (Temm.) (Nematoda: Trichuroidea). Ann. Acad. Brasil. Sci. 7 (4): 351-353. (Com. J.F. Teixeira de Freitas).

17. 1936. Estudo sobre o gênero Globocephalus Molin, 1861 (Nematoda: Strc ngyloidea). Mem. Inst. Oswaldo Cruz 31 (1): 69-79, 2 ests., 11 figs (Com. J.F. Teixeira de Freitas).

18. 1936. Estudo sobre os Capillarinae parasitos de mamíferos (Nematoda: Trichuroidea). Mem. Inst. Oswaldo Cruz 31 (1): 85-160, 16 ests., figs (Com. J.F. Teixceira de Freitas).

19. 1936. Novo parasito de Agouti paca (L.) (Nematoda: Strongyloidea). Mem. Inst. Oswaldo Cruz 31 (2): 357-359, 2 ests-. 7 figs (Com J.F. Teixeira de Freitas).

20. 1936. Notas e commentários sobre Triatomideos. Lista de espécies e sua distribuição geographica. Rev. Ent., Rio de Janeiro, 6 (2): 153-190 (Com Arthur Neiva).

21. 1936. O gênero Monopetalonema Diesing, 1861 (Nematoda: Filarioidea). Mem. Inst. Oswaldo Cruz 31 (4): 747-757, 3 ests., 12 figs (Com J.F. Teixeira de Freitas).

22. 1936. Sobre o Trichuris da nutria. Ann. Acad. Brasil. Sci. 8 (4): 319-322, 2 ests., 5 figs (Com J.F. Teixeira de Freitas).

23. 1936. Capillaria hepatica (Bancroft, 1893) em fígado de Sciurus aestuans L. Ann. Acad. Brasil. Sci. 8 (4): 323-325, figs 1-2 (Com J.F. Teixeira de Freitas).

24. 1937. Contribuição ao estudo do gênero Dirofilaria Railliet \& Henry, 1911. Mem. Inst. Oswaldo Cruz 32 (1): 37-54, ests. 1-7, 22 figs (Com J.F. Teixeira de Freitas).

25. Sobre um novo trematodeo parasito de Iguana tuberculata (Laur.). Mem. Inst. Oswaldo Cruz 32 (1): 55-58, figs 1-2 (Com J.F. Teixeira de Freitas).

26. 1937. Sur deux genres de Heligmosoninae (Nematoda: Strongyloidea). Ann. Acad. Brasil. Sci. 9 (1): 41-47, pls. 1-4, figs 1-11 and Papers on Helminthology published in commemoration of the $\mathbf{3 0}$ year Jubileum of Prof. Skrjabin, pp. 322-327, figs 1-11 (Com J.F. Teixeira de Freitas).

27. 1937. Pequena contribuição ao estudo da fauna helmintológica da Argentina 
(Nematoda). Mem. Inst. Oswaldo Cruz 32 (2): 195-209, ests. 1-13, 66 figs (Com J.F. Teixeira de Freitas \& J. Lins de Almeida).

28. 1937. Cimex limai Pinto, 1927, parasito de morcegos no Brasil. Mem. Inst. Oswaldo Cruz 32 (2): 211-215, 2 ests., 5 figs (Com M.C. Proença).

29. 1937. Nova Physaloptera parasita de marsupial (Nematoda: Spiruroidea). Mem. Inst. Oswaldo Cruz 32 (2): 221-223, 2 ests, 9 figs (Com J.F. Teixeira de Freitas).

30. 1937. Notas sobre Gongyloneminae Hall, 1916 (Nematoda: Spiruroidea). Mem. Inst. Oswaldo Cruz 32 (2): 299-304, 2 ests, 18 figs (Com J.F. Teixeira de Freitas).

31. 1937. Alguns helmintos da collecção de Pedro Severiano de Magalhães. Mem. Inst. Oswaldo Cruz 32 (2): 305-309, 1 est., 6 figs (Com J.F. Teixeira de Freitas).

32. 1937. Espécies de Capillaria em Cuba. Ann. Acad. Brasil. Sci. 9 (2): 91-97, ests. 1-6, figs 1-25 (Com J.F. Teixeira de Freitas).

33. 1937. Segunda espécie do gênero Filaria Mueller, 1787, s. str. Mem. Inst. Oswaldo Cruz 32 (3): 423-426, 1 est., 10 figs (Com J.F. Teixeira de Freitas).

34. 1937. Sobre Oswaldofilaria brevicaudata (Rhodain \& Vuylsteke, 1937), n.comb. (Nematoda: Filarioidea). Mem. Inst. Oswaldo Cruz 32 (3): 439-442, 2 ests., 10 figs (Com J.F. Teixeira de Freitas).

35.1937. Dilofilariose sub-cutânea dos cães no Brasil. Mem. Inst. Oswaldo Cruz 32 (3): 443-448, figs 1-4 (Com J. F. Teixeira de Freitas).

36. 1937. Pesquisas helmintológicas realizadas no Estado do Pará. I. Trematoda: Fascioloidea. Mem. Inst. Oswaldo Cruz 32 (3): 449-460, figs 1-12 (Com J.F. Teixeira de Freitas).

37. 1937. Novo trematodeo parasito de Strix flammea perlata (Licht.). Mem. Inst. Oswaldo Cruz 32 (4): 535-538, 2 ests., 5 figs (Com J.F. Teixeira de Freitas).

38. 1937. Sur une nouvèlle espèce du genre Viannaia Travassos, 1914. Papers of Helminthology published in commemoration of the 30 year Jubileum of Prof. Skrjabin, pp. 319-321, figs 1-8 (Com J.F. Teixeira de Freitas).

39. 1938. Três novos Trichostrongylideos parasitos de roedores brasileiros. Livr. Jub. Prof. Travassos, pp. 269-273, 3 ests., 19 figs (Com J.F. Teixeira de Freitas).

40. 1938. Pesquisas helminthológicas realizadas no Estado de Pará. II. Dois novos trematodeos de Caiman sclerops Gray. Mem. Inst. Oswaldo Cruz 33 (1): 53-56, 2 ests., 5 figs (Com J.F. Teixeira de Freitas).

41. 1938. Pesquisas helminthologicas realizadas no Estado do Pará. III. Um raro parasito das tartarugas fluviaes do Amazonas. Mem. Inst. Oswaldo Cruz 33 (1): 57-61, 2 ests., 5 figs (Com J.F. Teixeira de Freitas).

42. 1938. Sobre alguns trematodeos parasitos de Chelone mydas (L.), principalmente Paramphistomoidea. Mem. Inst. Oswaldo Cruz 33 (1): 79-87, 5 ests., 13 figs, 1 fig. texto (Com J.F. Teixeira de Freitas).

43. 1938. Aspiculuris caviellae, a new name for Aspiculuris schulzi Freitas, Lent \& Almeida, 1937, preoccupied. Proc. Helm. Soc. Wash. 5 (2): 65 (Com J.F. 
Teixeira de Freitas \& J. Lins de Almeida).

44. 1938. Pesquisas helminthologicas realizadas no Estado do Pará. IV. Trichostrongylideos de mamíferos. Mem. Inst. Oswaldo Cruz 33 (3): 363-380, 16 ests., 103 figs (Com J.F. Teixeira de Freitas).

45. 1938. Pesquisas helminthologicas realizadas no Estado do Pará. V. Gênero Diaphanocephalus Diesing, 1851 (Nematoda: Strongyloidea). Mem. Inst. Oswaldo Cruz 33 (3): 423-432, 7 ests., 24 figs (Com Teixeira de Freitas).

46. 1938. Pesquisas helminthologicas realizadas no Estado do Pará. VI. Acanthocephala. Mem. Inst. Oswaldo Cruz 33 (4): 455-459, 2 ests., 10 figs (Com J.F. Teixeira de Freitas).

47. 1938. Novo nematodeo parasito de rã sul-americana. Mem. Inst. Oswaldo Cruz 33 (4): 477-479, 3 ests., 13 figs (Com J.F. Teixeira de Freitas).

48. 1939. Novo gênero de helmintos parasitos de roedores (Nematoda: Filarioidea). Bol. Biol., n.s., 4 (1): 14-18, figs 1-2 (Com J.F. Teixeira de Freitas).

49. 1939. Pesquisas helmintológicas realizadas no Estado do Pará. VII. Trematoda. Paramphistomoidea. Bol. Biol., n.s., 4 (1): 82-86, 6 figs (Com J.F. Teixeira de Freitas).

50. 1939. Novo nematódeo parasito de pato doméstico (Spiruroidea). Bol. Biol., n.s., 4 (2): 177-180, figs 1-16 (Com J.F. Teixeira de Freitas).

51. 1939. Pesquisas helminthológicas. In: Relatório da excursão científica do Instituto Oswaldo Cruz realizada na zona da Estrada de Ferro Noroeste do Brasil, em Outubro de 1938. Bol. Biol., n.s., 4 (2): 221-249, 21 figs (Com Lauro Travassos \& J. F. Teixeira de Freitas).

52. 1939. Observações sobre a fauna de alguns ninhos de aves. In: Relatório da excursão científica do Instituto Oswaldo Cruz, realizada na zona da Estrada de Ferro Noroeste do Brasil, em Outubro de 1938. Bol. Biol., n.s., 4 (2): 258-260, 4 figs.

53. 1939. Revisão do gênero Catadiscus Cohn, 1904 (Trematoda, Paramphistomoidea). Bol. Biol., n.s., 4 (2): 305-315, figs 1-20 (Com J.F. Teixeira de Freitas).

54. 1939. Eutriatoma nigromaculata (Stal, 1872) n.comb., espécie venezuelana encontrada infestada pelo Schizotrypanum cruzi (Chagas, 1909) (Nota prévia). Brasil-Méd. 53 (27): 685-686, 1 fig. (Com Félix Pifano C.).

55. 1939. Novo caso de singamose em homem no Brasil. O Hospital, Rio de Janeiro, 16 (1): 111-118, figs 1-5 (Com Mauro Penna).

56. 1939. Some remarks on the genus Paraspidodera Travassos, 1914 (Nematoda: Subuluroidea). Vol. Jubil. Prof. Sadao Yoshida, Osaka, 2: 273-278 (Com J.F. Teixeira de Freitas).

57. Considerações sobre algumas espécies americanas do gênero Haematoloechus Looss, 1899 (Trematoda: Plagiorchoidea). Livr. Homen. Profs. A. e M. Ozório de Almeida, Rio de Janeiro, pp. 246-256, 1 fig. texto, 8 ests., 25 figs (Com J.F. Teixeira de Freitas).

58. 1939. Sobre o hematofagismo da Clerada apicicornis e outros artropodos; 
sua importância na transmissão da doença de Chagas. Mem. Inst. Oswaldo Cruz 34 (4): 583-606, figs,1-4.

59. 1939. Notas sobre triatomideos do Rio Grande do Sul e descrição de uma nova espécie. Mem. Inst. Oswaldo Cruz 34 (4): 607-610, figs 1-2 (Com Arthur Neiva e Cesar Pinto).

60. 1939. Dados experimentais sobre a infestação do Eutriatoma nigromaculata (Stal, 1872) pelo Schizotrypanum cruzi (Chagas, 1909), e sua redescrição. Mem. Inst. Oswaldo Cruz 34 (4): 627-635, figs 1-2 (Com Félix Pifano).

61. 1940. Sobre um novo triatomídeo chileno: Triatomaptera porteri. Brasil. Med. 54 (15): 265-267 (Com Arthur Neiva).

62. 1940. Arthur Neiva. In: Medicina no Brasil, Rio de Janeiro, pp 136-141. Rev. Ent. 11 (3): 954-960.

63. 1940. Estudos sobre Triatomídeos do Chile: Interessante caso de provável polimorfismo. Mem. Inst. Oswaldo Cruz 35 (2): 343-363, figs 1-6 (Com Arthur Neiva).

64. 1940. Histiostrongylus octacanthus n.sp. (Nematoda: Strongyloidea). Arq. Inst. Benjamin Baptista, Rio de Janeiro, 6: 91-96, ests. 1-3, figs 1-20 (Com J.F. Teixeira de Freitas).

65. 1940. Plagiorchis didelphidis (Parona, 1896), um parasito pouco conhecido de marsupiais. Arq. Inst. Benjamin Baptista 6: 131-135, figs 1-3 (Com J.F. Teixeira de Freitas).

66. 1940. Sur la position systématique de Distoma arrectum Molin, 1859. Ann. Acad. Brasil. Sci., 12(4): 319-323, 1 fig. Ann. Parasit. Hum. \& Comp. 20 (5/6): 279-283, fig. 1 (1944/45) (Com J.F. Teixeira de Freitas).

67. 1940. Sobre a identidade dos gêneros Panstrongylus Berg, 1879 e Mestor Kirkaldy, 1904. Redescrição de P. rufotuberculatus encontrado, na Venezuela, naturalmente infestado pelo Schizotrypanum cruzi. Rev. Ent., Rio de Janeiro, 11 (3): 629-639, figs 1-2 (Com Felix Pifano).

68. 1940. Estudos sobre os Triatomídeos do Estado de Minas Gerais, com descrição de uma espécie nova. Rev. Ent., Rio de Janeiro, 11 (3): 877-886, fig. 1, ests. 26-27, 4 figs (Com A. Vianna Martins).

69. 1941. Contribuição ao estudo dos gêneros Hedruris Nitzsch, 1821 (Nematoda). Papéis Avulsos Dep. Zool., Secr. Agric. S. Paulo 1 (16): 121-141., 2 ests., figs 1-11 (Com J. F. Teixeira de Freitas).

70. 1941. Estado atual de três espécies do gênero Cephalogonimus Poirier, 1886 (Trematoda). Mem. Inst. Oswaldo Cruz 35 (3): 515-524, figs 1-11 (Com J.F. Teixeira de Freitas).

71. 1941. Latrocimex spectans n.gen., n.sp., parasito de morcegos no Brasil (Hemiptera, Cimicidae). Rev. Brasil. Biol. 1 (1): 41-46, figs 1-4.

72. 1941. Contribuição ao conhecimento da sub-família Kathlaniinae Lane, 1914 (Nematoda: Subuluroidea). Arq. Zool. Est. S. Paulo 3 (2): 13-41, figs 1-2 (Com J.F. Teixeira de Freitas).

73. 1941. Sinopse dos Triatomídeos. Rev. Ent., Rio de Janeiro, 11 (1/2): 61-92, figs 1-28 (Com Arthur Neiva). 
74. 1941. Sobre um novo parasito de ariranha: Cloeoascaris longispiculum n.sp. (Nematoda, Ascaroidea). Rev. Brasil. Biol. 1 (3): 267-270, figs 1-13 (Com J.F. Teixeira de Freitas).

75. 1941. Sobre os filarídeos parasitos de lacertídeos neotrópicos. Rev. Brasil. Biol. 1 (4): 383-386, figs 1-10 (Com J.F. Teixeira de Freitas).

76. 1942. À propósito de Halltrema avitellina Lent \& Freitas, 1939. Rev. Brasil. Biol. 2 (1): 115-116, figs 1-4 (Com J.F. Teixeira de Freitas).

77. 1942. Nota sobre o encontro de cisticercos de Taenia solium L., 1758 com dois escólices. Rev. Brasil. Biol. 2 (2): 197-201, figs 1-7.

78. 1942. Estudos sobre os Triatomídeos do Estado do Rio Grande do Sul, com descrição de uma espécie nova. Rev. Brasil. Biol. 2 (2): 219-231, figs 1-33, 1 est.

79. 1942. Transmissores da moléstia de Chagas no Estado do Rio de Janeiro. Rev. Flum. Med. 7 (6): 151-161, 1 est.

80. 1942. Contribuição ao conhecimento dos Filarídeos de Dasipodídeos. Rev. Brasil. Biol. 2 (3): 275-280, figs 1-15 (Com J.F. Teixeira de Freitas).

81. 1942. Primeira espécie de Capillaria parașita de batráquio sulamericano (Nematoda, Trichuroidea). Rev. Brasil. Biol. 2 (3): 325-330, figs 1-4 (Com J.F. Teixeira de Freitas).

82. 1942. Sobre Dipetalonema spiralis (Molin, 1860) (Nematoda, Filarioidea). Mem. Inst. Oswaldo Cruz 37 (3): 383-389, figs 1-12 (Com J.F. Teixeira de Freitas).

83. 1943. Considerações sobre a validade do gênero Eutriatoma Pinto, 1926 (Hemiptera, Triatomidae). Rev. Brasil. Biol. 3 (2): 237-249, figs 1-8.

84. 1943. Triatomídeos da Guiana Holandesa. Redescrição de Panstrongylus lignarius (Walker, 1873). Mem. Inst. Oswaldo Cruz 38 (3): 485-496, figs 1-9.

85. 1943. Novo transmissor da doença de Chagas na cidade do Rio de Janeiro, D. F. Estudo dos gêneros Belminus Stal, 1859, Bolbodera Baldés,' 1910 e descrição de Parabelminus carioca n.g., n.sp. (Hemíptera, Triatomidae). Mem. Inst. Oswaldo Cruz 38 (3): 497-516, figs 1-10.

86. 1943. Arthur Neiva (Alocução proferida por ocasião da homenagem prestada pelo Instituto Oswaldo Cruz, no cemitério de S. João Baptista). Rev. Ent., Rio de Janeiro, 14 (1/2): 5-7.

87. 1943. Triatomídeos do Chile. Mem. Inst. Oswaldo Cruz 39 (1): 43-75, figs 1-6, 4 ests. col (Com Arthur Neiva).

88. 1943. In Memoriam - Arthur Neiva (1880-1943). Rev. Brasil. Biol. 3 (3): 273-291, foto.

89. 1944. Instituto de Higiêne de Assunção do Paraguai. Rev. Brasil. Biol. 4 (1): 131-137, figs 1-6.

90. 1944. Sobre uma nova espécie do gênero Chryxus Champion, 1898 (Chryxinae, Reduviidae, Hemiptera). Rev. Brasil Biol. 4 (2): 167-171, figs 1-19 (Com P. Wygodzinsky).

91. 1944. Nota preliminar sobre a ação do DDT (Dicloro-difenil-tricloroetano) em insetos transmissores da doença de Chagas. Rev. Brasil. Biol. 4 (3): 
329-331 (Com S.J. de Oliveira).

92. 1944. Nova espécie do gênero Aradomorpha Champion, 1899 (Hemiptera, Reduvioidea). Rev. Brasil. Biol. 4 (4): 475-481, figs 1-36 (Com Petr Wygodzinsky).

93. 1945. Nova espécie de Zelurus (Opisthacidius) da Amazônia (Reduviidae, Hemiptera). Rev. Brasil. Biol. 5 (1): 133-138, figs 1-6 (Com P. Wygodzinsky).

94. 1945. Os pareceres da Seção de Ciências Físicas e Naturais da Comissão Nacional do Livro Didático. A perícia na questão Potsch- Mello Leitão. Rio de Janeiro, Graf. St ${ }^{\circ}$ Antônio, 281p.

95. 1945. Trematódeos de morcegos colecionados no Paraguay. Rev. Brasil.

Biol. 5 (4): 499-507, figs 1-8 (Com J.F. Teixeira de Freitas).

96. 1946. Os pareceres da Seção de Ciências Físicas e Naturais da Comissão

Nacional do Livro Didático. O Perito e a Perícia no Processo Mello-Leitão.

Rio de Janeiro, Gráf. Aurora, 280p.

97. 1946. Contribuição ao conhecimento do gênero Zelurus Hahn (Spiniger auct.) (Reduviidae, Hemiptera). Mem. Inst. Oswaldo Cruz 43 (2): 205-270, figs 1-93 (Com P. Wygodzinsky).

98. 1946. Alguns helmintos de aves colecionadas no Paraguai. Mem. Inst. Oswaldo Cruz 43 (2): 271-285, figs 1-26 (Com J.F. Teixeira de Freitas \& M. Cavalcanti Proença).

99. 1946. Um nuevo Cimicidae encontrado en nidos de Myiopsitta monacha cotorra (Vieillot). Anal. Inst. Med. Reg., Tucuman, 1 (3): 337-348, figs 1-17 (Com J.W. Abalos).

100. 1946. Infestação de apaiarís Astronotus ocellatus (Agassiz) pelo nematódeo Goezia spinulosa (Diesing, 1839). Rev. Brasil. Biol. 6 (2): 215-222, figs 1-16 (Com J.F. Teixeira de Freitas).

101. 1946. Porrocaecum sulcatum (Rudolphi, 1819) (Nematoda, Ascaroidea).

Rev. Brasil. de Biol. 6 (2): 235-238, figs 1-13 (Com J.F. Teixeira de Freitas). 102. 1946. Alguns helmintos de batráquios colecionados no Paraguai. Mem. Inst.

Oswaldo Cruz 44 (1): 195-214, figs 1-56 (Com J.F. Teixeira de Freitas \& M. Cavalcanti Proença).

103. 1946. Novo hemíptero hematófago do gênero Panstrongylus Berg, 1879. Rev.

Brasil. Biol. 6 (4): 459-465, figs 1-19 (Com Cesar Pinto)

104. 1946. Alguns nematodos de murciélagos coleccionados en el Paraguay. Rev.

Brasil. Biol., 6 (4): 485-497, figs 1-46 (Com J.F. Teixeira de Freitas \& M.

Cavalcanti Proença).

105. 1947. Sobre algumas espécies de Zelurus Hahn (Reduviidae, Hemiptera).

Rev. Brasil. Biol. 7 (1): 25-55, figs 1-42 (Com P. Wygodzinsky).

106. 1947. Revisão da sub-família Deletrocephalinae Railliet, 1916 (Nematoda,

Strongyloidea). Rev. Brasil. Biol. 7 (1): 73-100, figs 1-83 (Com J.F. Teixeira de Freitas).

107. 1947. Contribuição ao conhecimento dos Reduviinae americanos (Reduviidae, Hemiptera). Rev. Brasil. Biol. 7 (3): 341-368, figs 1-88 (Com P. Wygodzinsky). 
108. 1947. Notes on some assassin bugs of the genus Zelurus from the collections of the United States National Museum. Proc. U. S. Nat. Mus. 97 (3217): 343-349, pls. 4-7, figs 1-28 (Com P. Wygodzinsky).

109. 1948. On two new genera of American Reduviinae, with a key and notes on others (Reduviidae, Hemiptera). Rev. Brasil. Biol. 8 (1): 43-55, figs 1-54 (Com P. Wygodzinsky).

110. 1948. Spiruroidea parasitos de Rheiformes (Nematoda). Mem. Inst. Oswaldo Cruz 45 (4): 743-760, ests. 1-10, figs 1-49 (Com J.F. Teixeira de Freitas).

111. 1948. O gênero Rhodnius Stal, 1859 (Hemiptera, Reduviidae). Rev. Brasil. Biol. 8 (3): 297-339, figs 1-47.

112. 1948. Uma coleção de Nematódeos, parasitos de vertebrados, do Museu de História Natural de Montevidéo. Mem. Inst. Oswaldo Cruz 46 (1): 1-71, figs 1-136 (Com J.F. Teixeira de Freitas).

113. 1948. Triatoma and Chagas' Disease. Proc. 4th Int. Congr. Trop. Med. \& Malaria, Washington, 2: 1690-1701, figs 1-5.

114. 1949. Nova Dirofilaria parasita do aparelho circulatório da ariranha (Nematoda, Filarioidea). Rev. Brasil. Biol. 9 (3): 377-380, figs 1-12 (Com J.F. Teixeira de Freitas).

115. 1950. Nova espécie de Triatoma Laporte, 1833 (Hemiptera, Reduviidae).

Rev. Brasil. Biol. 10 (4): 437-440, figs 1-4.

116. 1951. Estudos sobre o gênero Zelurus Hahn (Reduviidae, Hemiptera). Rev.

Brasil. Biol. 11 (1): 1-28, figs 1-43 (Com P. Wygodzinky).

117. 1951. Segunda espécie do gênero Bolbodera Valdés, 1910 (Hemiptera, Reduviidae, Triatominae). Rev. Brasil. Biol. 11 (2): 153-156, figs 1-3.

118. 1951. Espécies do gênero Zelurus Hahn (Hemiptera, Reduviidae). Rev. Brasil. Biol. 11 (2): 173-179, figs 1-14 (Com P. Wygodzinsky).

119. 1951. Contribuição ao conhecimento de Zelurus fulvomaculatus (Berg, 1879) e sete outras espécies afins (Reduviidae, Hemiptera). Mem. Inst. Oswaldo Cruz 49: 575-594, figs 1-20 (Com. P. Wygodzinsky).

120. 1951. Novo Triatoma do Estado de Minas Gerais (Brasil) (Hemiptera, Reduviidae). Rev. Ent., Rio de Janeiro, 22 (1-3): 349-352, figs 1-3.

121. 1951. Acarinos das vias respiratórias do canário (Serinus canarius L.) por Sternostoma tracheacolum Lawrence, 1948. Rev. Brasil. Biol. 11 (4): 399-406, figs 1-7 (Com C. Magarinos Torres \& L. F. Moreira).

122. 1951. Triatominae das regiões oriental, australiana, etíopica e paleártica, com descrição de uma nova espécie (Hemiptera, Reduviidae). Rev. Brasil. Biol. 11 (4): 423-429, figs 1-3.

123. 1951. Triatoma longipes Barber, 1937, um sinônimo de Triatoma recurva (Stal, 1868) (Hemiptera, Reduviidae). Ciência, México, 11 (5-6): 156-158, figs 1-3.

124. 1953. Um novo hemiptero hematófago da Venezuela (Reduviidae, Triatominae). Rev. Brasil. Biol. 13 (2): 169-172, figs 1-3.

125. 1953. Triatoma recurva (Stal, 1868) e seu sinônimo Triatoma longipes 
Barber, 1937 (Hemiptera, Reduviidae). Arq. Mus. Nac., Rio de Janeiro, 42: 261-264, 3 figs

126. 1953. Nova espécie de Triatoma da região oriental (Hemiptera, Reduviidae). Rev. Brasil. Biol. 13 (4): 315-319, figs 1-4.

127. 1954. Comentários sobre o gênero Rhodnius Stal, com descrição de uma nova espécie do Brasil (Hemiptera, Reduviidae). Rev. Brasil. Biol. 14 (3): 237-247, figs 1-20.

128. 1954. Contribuição ao conhecimento das espécies de Zelurus do grupo femoralis (Reduviidae, Hemiptera). Rev. Brasil. Biol. 14 (4): 407-442, figs 1-46 (Com P. Wygodzinsky).

129. 1954. Contribuición al conocimento del gênero Belminus Stal, 1859 (Triatominae, Reduviidae, Hemiptera). Anal. Inst. Med. Reg., Tucumán, 4 (1): 85-105, figs 1-48 (Com A. Herrer \& P. Wygodzinsky).

130. 1955. Espécies bolivianas dos gêneros Zelurus Hahn e Opisthacidius Berg (Reduviidae, Hemiptera). Rev. Brasil. Biol. 15 (1): 103-110, figs 1-12 (Com P. Wygodzinsky).

131. 1955. Espécies de Zelurus Hahn e Opisthacidus Berg de Colômbia, Equador e Peru (Reduviidae, Hemiptera). Mem. Inst. Oswaldo Cruz 53 (1): 135-142, figs 1-12 (Com P. Wygodzinsky).

132. 1955. Revisão dos Piratinae americanos. I: O gênero Tydides Stal, 1865 (Hemiptera, Reduviidae). Rev. Brasil. Biol. 15 (2): 159-176, figs 1-36.

133. 1955. Espécies venezuelanas dos gêneros Zelurus Hahn e Opistha cidius

Berg (Reduviidae, Hemiptera). Rev. Brasil. Biol. 15 (2): 177-190, figs 1-27

(Com P. Wygodzinsky).

134. 1956. Microtomus pessoai sp.n. da Venezuela (Hemiptera, Reduviidae).

Rev. Brasil. Biol. 16 (1): 77-80, figs 1-3 (Com Miguel A. Suarez).

135. 1956. Situação atual do gênero Opisthacidius Berg, 1879 (Hemiptera, Reduviidae). Rev. Brasil. Biol. 16 (3): 327-334, figs 1-10 (Com P. Wygodzinsky).

135A. 1956. Adolpho Lutz (Homenagem no centenário do seu nascimento).

Ciência e Cultura 8 (1): 50-51.

136. 1957. Notas sobre Zelurus Hahn (Hemiptera, Reduviidae). Rev. Brasil.

Biol. 17 (1): 21-42, figs 1-23 (Com P. Wygodzinsky).

137. 1957. Problemas bibliográficos dos cientistas e pesquisadores. Ciência e Cultura 8 (4): 247-248.

138. 1958. Um novo Rhodnius Stal do Equador (Hemiptera, Reduviidae). Rev.

Brasil. Biol. 18 (2): 181-185, figs 1-5 (Com Luis A. Leon).

139. 1958. Notes on an interesting structure of the pygophore of Rhodnius Stal, 1859 (Hemiptera, Reduviidae). Rev. Brasil. Biol. 18 (4): 381-383, figs 1-10.

140. 1959. Sobre los géneros Pseudozelurus Lent \& Wygod. y Zeluroides Lent \&

Wygod. (Reduviinae, Reduviidae, Hemiptera). Rev. Brasil. Biol. 19 (4): 351-365, figs 1-26 (Com P. Wygodzinsky).

141. 1960. Sobre dois pretensos reduvídeos hematófagos africanos do gênero Panstrongylus Berg, 1879 (Reduviidae, Triatominae). Rev. Brasil. Biol. 20 
(2): 163-170, figs 1-4.

142. 1961. The tropicopolita spider Uloborus geniculatus (Olivier), a new predator of the cigarette beetle in warehouses; possibility of its use in biological control. Rev. Brasil. Biol. 21 (4): 351-358, figs 1-7 (Com S.J. de Oliveira).

143. 1962. Estado atual dos estudos sobre os transmissores da doença de Chagas (Relatório). Anais Congr. Intern. Doença de Chagas, Rio de Janeiro, 3: 739-760, figs 1-14.

144. 1962. Preliminary notes on Chironomid (Diptera) intersexuality induced by Agemomermis (Nematoda). Rev. Brasil. Biol. 22 (4): 357-365, figs 1-18(Com S.J. Oliveira).

145. 1964. Uloborus geniculatus (Oliv.), aranha predadora da broca do fumo armazenado Lasioderma serricome (F.), e a possibilidade do seu uso no controle biológico. Rev. Brasil. Biol. 24 (4): 461-463, 1 fig. (Com S.J. de Oliveira).

146. 1965. Contribuição ao conhecimento dos Phloeidae Dallas, 1851, com um estudo sobre genitália (Hemiptera, Pentatomoidea). Rev. Brasil. Biol.25 (2): 123-144, figs 1-55 (Com José Jurberg).

147. 1965. O gênero Psammolestes Bergroth, 1911, com um estudo sobre a genitália das espécies (Hemiptera, Reduviidae, Triatominae). Rev. Brasil. Biol. 25 (4): 349-376, figs 1-78 (Com José Jurberg).

148. 1966. Os estádios larvares de Phloeophana longirostris (Spinola, 1837) (Hemiptera, Pentatomoidea). Rev. Brasil. Biol. 26 (1): 1-4, figs 1-10 (Com José Jurberg).

149. 1966. Os tipos de G. Breddin de Spiniger (Zelurus Hahn) (Hemiptera, Reduviidae). Rev. Brasil. Biol. 26 (2): 145-164, figs 1-40 (Com P. Wygodzinsky).

150. 1966. Revisão dos Piratinae americanos. II: O gênero Phorastes Kirkaldy, 1900, com um estudo sobre a genitália das espécies (Hemiptera, Reduviidae). Rev. Brasil. Biol. 26 (3): 297-314, figs 1-54 (Com José Jurberg). 151. 1966. Walter Oswaldo Cruz, sucesso e fracasso de um cientista (Alocução proferida na sessão de homenagem realizada em 11 de abril de 1967 na Academia Brasileira de Ciências). 16p., Rio de Janeiro, GB. Também em Rev. Civil Brasil. 16; e Ciência e Cultura 19 (4): 712-717.

152. 1967. Revisão dos Piratinae americanos. III. As espécies do gênero Tydides Stal, com um estudo sobre a genitália (Hemiptera, Reduviidae). Atas do Simpósio sobre a Biota Amazônica 5: 337-363, figs 1-82 (Com José Jurberg).

153. 1967. Zoologia. In: Anuário 1967 da Enciclopédia Delta-Larousse. Acontecimentos de 1966 ( $C f$. pp. 592-599, figs). Rio de Janeiro, Editora Delta S.A.

154. 1967. Algumas informaçōes sobre Triatoma spinolai Porter, 1934, com um estudo sobre as genitálias externas (Hemiptera, Reduviidae). Rev. Brasil. Biol. 27 (3): 273-288, figs 1-42 (Com José Jurgerg).

155. 1968. Situação atual das espécies de "Zelurus" Hahn do grupo "formosus" (Hemiptera, Reduviidae). Rev. Brasil. Biol. 28 (3): 317-326, figs 1-18 (Com 
P. Wygodzinsky).

156. 1968. Zoologia. In: Anuário 1968 da Enciclopédia Delta-Larousse. Acontecimentos de 1967 ( $C f$. pp. 600-605 figs). Rio de Janeiro, Editora Delta S.A.

157. 1968. Estudo morfológico comparativo de Panstrongylus geniculatus (Latreille, 1811) e Panstrongylus megistus (Burmeister, 1835) e suas genitálias externas (Hemiptera, Reduviidae, Triatominae). Rev. Brasil. Biol. 28 (4): 499-520, figs 1-72 (Com José Jurberg).

158. 1969. Observaçōes sobre o ciclo evolutivo, em laboratório, do Panstrongylus geniculatus (Latreille, 1811) (Helmiptera, Reduviidae, Triatominae). An. Acad. Brasil. Ciênc. 41 (1): 125-131, figs 1-7 (Com José Jurberg).

159. 1969. O gênero Cavernicola Barber, 1937, com um estudo sobre a genitália externa (Hemiptera, Reduviidae, Triatominae). Rev. Brasil. Biol. 29 (3): 317-327, figs 1-28 (Com José Jurberg).

160. 1969. O gênero Rhodnius Stal, 1859, com um estudo sobre a genitália das espécies (Hemiptera, Reduviidae, Triatominae). Rev. Brasil. Biol. 29 (4): 487-560, figs 1-219, est. 1 col (Com José Jurberg).

161. 1970. Insect juvenile hormone-like activity in a diterpene. Rev. Brasil. Biol. 30 (1): 55-60, figs 1-3 (Com H. Moussatché, M. Kitagawa \& B. Gilbert).

162. 1970. O gênero Eratyrus Stal, 1859, com um estudo sobre a genitália externa (Hemiptera, Reduviidae, Triatominae). Rev. Brasil. Biol. 30 (2): 297-312, figs 1-64 (Com José Jurberg).

163. 1970. Observações sobre o ciclo evolutivo, em laboratório, do Rhodnius robustus Larrousse, 1927 (Hemiptera, Reduviidae, Triatominae). Rev. Brasil. Biol. 30 (3): 477-481, figs 1-15 (Com José Jurberg e Vera R.G. Reis). 164. 1971. O gênero Paratriatoma Barber, 1938, com um estudo sobre a genitália externa (Hemiptera, Reduviidae, Triatominae). Rev. Brasil. Biol. 31 (1): 39-48, figs 1-23 (Com José Jurberg).

165. 1971. Estudo morfológico comparativo de duas espécies do gênero Leptoglossus Guerin, 1838 e suas genitálias (Hemiptera, Coreidae). Rev. Brasil. Biol.31 (4): 453-465, figs 1-76 ((Com José Jurberg e Vera R.G. Reis).

166. 1972. O gênero Dipetalogaster Usinger, 1939, com um estudo sobre a genitália externa (Hemiptera, Reduviidae, Triatominae). Studia Entomológica 15: 465-484, figs 1-49 (Com José Jurberg).

167. 1972. Desenvolvimento da entomologia no Instituto Oswaldo Cruz. Discurso na Academia Brasileira de Ciência, ao receber o Prêmio Costa Lima. Ciência e Cultura 24 (12): 1192-1200.

168. 1973. Hallazgo en Venezuela del triatomino Rhodnius robustus Larrousse, 1927 en la palma Attalea maracaibensis Martius (Hemiptera, Reduviidae). Bol. Inform. Dir. Malariol. Saneam. Amb., Maracay, Venezuela, 13 (5/6): 175-179, figs 1-3 (Com Alberto Valderrama).

169. 1975. O gênero Panstrongylus Berg, 1879, com um estudo sobre a genitália externa das espécies (Hemiptera, Reduviidae, Triatominae). Rev. Brasil. Biol. 35 (3): 379-438, figs 1-241 (Com José Jurberg).

170. 1977. Observações, em laboratório, sobre o ciclo evolutivo de Rhodnius 
prolixus Stal, 1859, Rhodnius pictipes Stal, 1872 e Rhodnius neivai Lent, 1953 (Hemiptera, Reduviidae, Triatominae). Rev. Brasil. Biol. 37 (2): 325-344, figs 1-28 (Com Alberto Valderrama)

171. 1977. As ninfas do $5^{\circ}$ estádio de Phimophorus spissicomis Bergroth, 1886 (Hemiptera, Reduviidae). Rev. Brasil. Biol. 37 (3): 521-524, figs 1-9 (Com José Jurberg).

172. 1978. Estudo comparativo da genitália externa masculina de seis espécies de Triatoma Lap., 1832 que mais frequentemente habitam o domicilio humano no Brasil. Rev. Brasil. Biol. 38 (4): 931-944, figs 1-62 (Com José Jurberg). 173. 1978. O massacre de Manguinhos. Coleção Depoimentos. Rio de Janeiro, Avenir Editora, 70p.

174. 1979. Ciência e Universidade. Espaço, Cadernos de Cultura, USU, 1 (1): 17-23.

175. 1979. Revision of the Triatominae (Hemiptera, Reduviidae), and their significance as vectors of Chagas' disease. Bull. Amer. Mus. Nat. Hist. 163 (3): 125-520, figs 1-320 (Com P. Wygodzinsky).

176. 1980. Comentários sobre a genitália externa masculina em Triatoma Laporte, 1832 (Hemiptera, Reduviidae). Rev. Brasil. Biol. 40 (3): 611-627, figs 1-80 (Com José Jurberg).

177. 1980. Description of one new monotypic genus of Reduviidae from Panama and two from Southern India (Hemiptera, Reduviidae). Rev. Brasil. Biol. 40 (4): 733-742, figs 1-5 (Com P. Wygodzinsky).

178. 1980. Arthur Neiva - Vida e Obra. Conferência proferida em 21 de março de 1980, no Instituto Oswaldo Cruz, em comemoração ao centenário de nascimento do Dr. Arthur Neiva. Ciência e Cultura 32 (11): 1581-1587.

179. 1981. As espécies insulares de Cuba do gênero Triatoma Laporte (Hemiptera, Reduviidae). Rev. Brasil. Biol. 41 (2): 431-439, figs 1-26 (Com José Jurberg).

180. 1981. Ciência Pura x Aplicada. As aspiraçōes e o modo de trabalho do cientista. In: Administração da atividade científica. Brasília, FINEP/CNPq (Coleção Estudos de Política Científica e Tecnológica, vol. 5 (Cf. pp. 43-56). 181. 1982. A genitália externa dos machos no complexo Triatoma phyllosoma, espécies mexicanas transmissoras da doença de Chagas. Mem. Inst. Oswaldo Cruz 77 (3): 299-317, figs 1-67 (Com José Jurberg).

182. 1982. Eles transmitem a doença de Chagas. Ciência Hoje, Rio de Janeiro, 1 (2): 45-49, figs

183. 1982. Microtriatoma pratai Sherlock \& Guitton, 1982 é sinônimo do hemíptero predador Aradomorpha championi Lent \& Wygodzinsky, 1944 (Reduviidae, Reduviinae). Mem. Inst. Oswaldo Cruz 77 (4): 449-451.

184. 1983. Ministério da Ciência, solução possível? Ciência e Cultura 35 (8): 1106-1111.

185. 1983. Estudo comparativo da genitália externa masculina de Linshcosteus costalis Ghauri e L. kali Lent \& Wygodzinsky (Hemiptera, Reduviidae, Triatominae). Mem. Inst. Oswaldo Cruz 78 (4): 421-429, figs 1-17 (Com José 
Jurberg).

186. 1984. A genitália externa masculina na tribu Bolboderini (Hemiptera, Reduviidae, Triatominae). Mem. Inst. Oswaldo Cruz 79 (1): 1-27, figs 1-62 (Com José Jurberg).

187. 1985. Sobre a variação infra-específica em Triatoma dimidiata (Latreille) e Triatoma infestans (Klug) (Hemiptera, Reduviidae). Mem. Inst. Oswaldo Cruz. 80 (3): 285-299, figs 1-19 (Com José Jurberg).

188. 1986. A genitália externa dos machos em quatro espécies do gênero Sirthenea Spinola, 1837 (Hemiptera, Reduviidae, Piratinae). Mem. Inst. Oswaldo Cruz 81 (3): 273-292, figs 1-52 (Com Vânia Maria Nunes Victório e José Jurberg).

189.1987. A genitália externa dos machos de sete espécies de Triatoma Laporte, 1832 da região neártica (Hemiptera, Reduviidae). Mem. Inst. Oswaldo Cruz 82 (2): 227-246, figs 1-75 (Com José Jurberg).

190. 1987. Estudo anatômico e morfométrico dos folículos testicualres de algumas espécies de Triatominae (Hemiptera, Reduviidae). Mem. Inst. Oswaldo Cruz 82 (4): 543-550, figs 1-3 (Com Teresa Cristina M. Gonçalves e Josimar Ribeiro de Almeida).

191. 1989. Estudo comparativo da genitália externa masculina de duas espécies de Zelurus Hahn, 1826 (Hemiptera, Reduviidae, Reduviinae). Mem. Instituto Oswaldo Cruz 84 (2): 219-226, figs 1-34 (Com Vânia Maria Nunes Victório e José Jurberg).

192. 1989. Estudos da genitália externa masculina de duas espécies de Opisthacidius Berg, 1879 (Hemiptera, Reduviidae, Reduviinae). Mem. Inst. Oswaldo Cruz 84 (Supl. IV): 319-326, figs 1-25 (Com José Jurberg).

193. 1989. Acanthocephalus ula $\mathrm{n}$.sp. (Acanthocephala, Echinorhynchidae) and

Schulzia usu n.sp. (Nematoda, Molineidae) described from At lopus oxyrhynchus (Amphibia) from Venezuela. Rev. Brasil. Biol. 4) (4): 1085-1091, figs 1-13 (Com Claudia Portes Santos).

194. 1990. The genus Aspidodera Railliet and Henry, 1912 (Nematoda: Heterakoidea): Revision, new synonyms and key for species. Rev. Brasil. Biol. 50 (4): 1017-1031, figs 1-35 (Com Claudia Portes Santos e Delir Corrêa Gomes).

195. No prelo. Estudo comparativo da genitália externa masculina de Triatoma neotomae Neiva, 1911 e Triatoma nitida Usinger, 1939 (Hemiptera, Reduviidae). Mem. Inst. Oswaldo Cruz.

Recebido em 31.V.1992; aceito em 25.XI.1993. 